\title{
Response to "Does amantadine have a protective effect against COVID-19?"
}

\author{
Philip W. Tipton, Zbigniew K. Wszolek \\ Department of Neurology, Mayo Clinic, Jacksonville, Florida, USA
}

Information about SARS-CoV-2 is accumulating at an unprecedented rate. Any hope of returning to a pre-COVID way of life seems to rest on the shoulders of researchers tasked with developing effective treatments and a vaccine.

This has generated an expectation for science to move faster than ever before. Evidence of acute infection can be assessed in a matter of days; however, the long- term effects may require years before reaching the threshold for clinical detection. Long-term effects are also subject to more confounders. Therefore, while acute care investigators are illuminating immediate viral effects, those studying neurodegeneration are left to ponder and to speculate about the downstream effects of viral exposure. There is now a reemerging focus on a possible link between SARS-CoV-2 and neurodegenerative diseases, specifically Parkinson's Disease (PD) [1].

Many studies across the globe have published COVID-19 patient characteristics and comorbidities to help us understand which people are more susceptible to infection and who is likely to have a more severe disease course. One of the largest studies looked at the characteristics of 5,700 patients hospitalised with COVID-19 [2]. Older individuals trended towards poorer outcomes, which is consistent with current thinking. Comorbidities with the largest representation included cancer, cardiovascular disease, chronic respiratory disease, immunosuppression, and others.

PD affects over $1 \%$ of the population over the age of 60 , and $5 \%$ of those older than 85 [3]. Moreover, PD is the second most common neurodegenerative disease behind Alzheimer's Disease $(A D)$, yet neither disease was represented in this cohort, which had a median age of 63. Perhaps there are protective factors in these otherwise vulnerable populations that seem to make them disproportionately less affected. It is also possible that medications used to treat the symptoms of $\mathrm{PD}$ and $\mathrm{AD}$ are effective against SARS-CoV-2.

In our Letter to the Editors of Neurologia i Neurochirurgia Polska, we hypothesised that some adamantane derivatives used in neurodegenerative populations may play a protective role against SARS-CoV-2 [4]. These include amantadine, commonly used to treat patients with $\mathrm{PD}$, and memantine, which is commonly used in dementing illnesses such as AD.

Our hypothesis was largely based on evidence that similar medications are efficacious against other coronaviruses [5]. Given the newness of SARS-CoV-2, this hypothesis is un-tested and there were no randomised control trials at the time. To date, there still have been no direct trials; however, supportive evidence is emerging. In this issue, Cortés Borra responds to our Letter to the Editors, and describes a 75-year-old woman with a longstanding history of PD treated with amantadine, among other medications [6]. Unfortunately, the woman's husband died from COVID-19 pneumonia. Despite an almost certain SARS-CoV-2 exposure from direct contact with her husband, COVID-19 PCR testing was negative, and she remained symptom-free.

Even though anecdotal evidence like this must always be interpreted with extreme caution, Cortés Borra's Commentary appears to support our hypothesis. Adding to this is a recently published study that aimed at determining whether patients with PD were at greater risk of COVID-19 [7]. While the study found no significant difference from the general population, the authors showed that vitamin D supplementation was associated with lower rates of infection in patients with PD. We find it interesting that there was a relative risk reduction of $100 \%$ among PD patients on amantadine. This was not statistically significant, and absolute risk reduction was only $1 \%$; however, only $2 \%$ of $\mathrm{PD}$ patients were taking amantadine. Rejdak and Grieb found that 5/5 PD patients on amantadine tested positive for SARS-CoV-2 but experienced no symptoms after $>14$ days [8]. Similarly, 7/7 patients on memantine had asymptomatic SARS-CoV-2 infections.

Reports vary, but this asymptomatic rate is much higher than a recent report of $56 \%$ among nursing home residents where only $3 \%$ remained asymptomatic one week after testing [9]. 
We find this difference in symptomatic rates to be remarkable given that elderly individuals are generally considered to be 'high risk'.

It could be that the high risk of the PD population goes beyond that of older age, and may be tied to viral neurotropism. Validating this will require well-designed studies with years of follow up. However, an adequately powered retrospective study would be sufficient to disprove our hypothesis. We feel this study should be conducted and, if our hypothesis stands, be followed by prospective validation.

We congratulate Cortés Borra on his contribution and hope that others will make further progress in this field and thus protect our patients.

\section{Acknowledgements}

Mayo Clinic is an American Parkinson's Disease Association (APDA) Information and Referral Centre and APDA Centre for Advanced Research.

\section{Financial disclosure/Conflicts of interest:}

Dr. Tipton reports no disclosure or conflict of interest.

Dr. Wszolek receives support from the Mayo Clinic Centre for Regenerative Medicine, and gifts from The Sol Goldman Charitable Trust, and the Donald G. and Jodi P. Heeringa Family, the Haworth Family Professorship in Neurodegenerative Diseases fund, and The Albertson Parkinson's Research Foundation. He serves as PI or Co-PI on Biogen, Inc. (228PD201), and BioHaven Pharmaceuticals, Inc. (BHV4157206 and BHV3241-301) grants. He serves as PI of the Mayo Clinic American Parkinson's Disease Association (APDA) Information and Referral Centre. He is a co-editor-in-chief of Neurologia i Neurochirurgia Polska (the Polish Journal of Neurology and Neurosurgery).

Funding sources for the study: None

Search terms: COVID-19, amantadine, memantine, Parkinson's Disease

Author roles: Dr. Tipton: manuscript composition and formatting; Dr. Wszolek: manuscript editing.
Glossary

$\mathrm{AD}$ - Alzheimer's Disease

COVID-19 - coronavirus 19

PD - Parkinson's Disease

SARS-CoV-2 - Severe Acute Respiratory Syndrome Coronavirus 2

\section{References}

1. Lippi A, Domingues R, Setz C, et al. SARS-CoV-2: At the Crossroad Between Aging and Neurodegeneration. Mov Disord. 2020; 35(5): 716 720, doi: 10.1002/mds.28084, indexed in Pubmed: 32291797.

2. Richardson S, Hirsch JS, Narasimhan M, et al. and the Northwell COVID-19 Research Consortium. Presenting Characteristics, Comorbidities, and Outcomes Among 5700 Patients Hospitalized With COVID-19 in the New York City Area. JAMA. 2020 [Epub ahead of print], doi: 10.1001/jama.2020.6775, indexed in Pubmed: 32320003.

3. Reeve A, Simcox E, Turnbull D. Ageing and Parkinson's disease: why is advancing age the biggest risk factor? Ageing Res Rev. 2014; 14: 19-30, doi: 10.1016/j.arr.2014.01.004, indexed in Pubmed: 24503004.

4. Tipton PW, Wszolek ZK. What can Parkinson's disease teach us about COVID-19? Neurol Neurochir Pol. 2020; 54(2): 204-206, doi: 10.5603/PJNNS.a2020.0039, indexed in Pubmed: 32323862.

5. Brison $\mathrm{E}$, Jacomy $\mathrm{H}$, Desforges $\mathrm{M}$, et al. Novel treatment with neuroprotective and antiviral properties against a neuroinvasive human respiratory virus. J Virol. 2014; 88(3): 1548-1563, doi: 10.1128/ JVI.02972-13, indexed in Pubmed: 24227863.

6. Cortés Borra A. Does amantadine have a protective effect against COVID-19? Neurol Neurochir Pol. 2020 [Epub ahead of print], doi: 10.5603/PJNNS.a2020.0041, indexed in Pubmed: 32495926.

7. Fasano A, Cereda E, Barichella M, et al. COVID-19 in Parkinson's Disease Patients Living in Lombardy, Italy. Mov Disord. 2020 [Epub ahead of print], doi: 10.1002/mds.28176, indexed in Pubmed: 32484584.

8. Rejdak K, Grieb P. Adamantanes might be protective from COVID-19 in patients with neurological diseases: multiple sclerosis, parkinsonism and cognitive impairment. Mult Scler Relat Disord. 2020 [Epub ahead of print]; 42: 102163, doi: 10.1016/j.msard.2020.102163, indexed in Pubmed: 32388458.

9. Arons MM, Hatfield KM, Reddy SC, et al. Public Health-Seattle and King County and CDC COVID-19 Investigation Team. Presymptomatic SARS-CoV-2 Infections and Transmission in a Skilled Nursing Facility. N Engl J Med. 2020; 382(22): 2081-2090, doi: 10.1056/NEJMoa2008457, indexed in Pubmed: 32329971. 